\title{
Novel insights regarding the sigmoidal pattern of resistance to neomycin conferred by the aphll gene, in Streptomyces lividans
}

\author{
Nicolas Seghezzi ${ }^{1}$, Marie-Joelle Virolle ${ }^{1 *}$ and Patrick Amar ${ }^{2^{*}}$
}

\begin{abstract}
A library of synthetic promoters of various strengths, specifically constructed for Streptomyces species, was cloned in the promoter-probe plasmid pIJ487, upstream of the promoter-less aphll gene that confers resistance to neomycin. The survival rates conferred by promoters were assessed in the presence of $100 \mu \mathrm{g} \cdot \mathrm{ml}^{-1}$ neomycin. The correlation between the transcriptional activity of the aphll gene (estimated by RT-PCR) and the resistance to neomycin (expressed as survival rate) indicated a sigmoid rather than a linear correlation. In this issue, we propose a tentative explanation for this sigmoidal pattern of resistance in relation with the level of aphll gene expression. Beyond this specific example, our model might constitute a sound explanation for the generally observed but never explained sigmoidal shape of classical inhibition curves obtained in the presence of linearly increasing antibiotic concentrations.
\end{abstract}

Keywords: Bacteria, Gene expression/regulation, Growth and survival, Modelling

\section{Introduction}

Antibiotics have been the most useful therapeutic agents of the twentieth century (Levy 2002). However, more and more bacteria have developed resistance to all existing antibiotics and antimicrobial resistance was recently recognized as one of the greatest threats to human health (Gyssens 2011). Indeed an increasing number of patients suffer from serious life-threatening antimicrobial-resistant infections against which only very few, if any, effective antibiotics are available (Levy 1998). Alarmingly, as the number of patients dying from antibiotic-resistant infections rises, the number of new antibiotics in development is plummeting (Butler and Cooper 2012; Mahajan and Balachandran 2012). The antibiotic resistance genes acquired by human pathogens are thought to originate from micro-organisms of the environment including Streptomyces (Forsberg et al. 2012). These bacteria are antibiotic producers and thus contain the corresponding antibiotic resistance genes for self protection (Allen et al. 2010; Davies and Davies 2010;

\footnotetext{
* Correspondence: marie-joelle.virolle@igmors.u-psud.fr; pa@lri.fr ${ }^{1}$ Institut de Génétique et Microbiologie, UMR8621 CNRS Université Paris-Sud, Orsay 91405, France

${ }^{2}$ Laboratoire de Recherche en Informatique, Université Paris-Sud, UMR8623 CNRS, and INRIA Saclay, Orsay 91405, France
}

Nikaido 2009). The genes conferring resistance to antibiotics are spreading by horizontal transfer in the microbial population and the release of antimicrobials in the environment likely selects micro-organisms carrying these genes (Alonso et al. 2001; Martinez 2008). The mechanisms conferring antibiotic resistance in micro-organisms include enzymatic inactivation or modification of the antibiotic, modification of host targets to prevent antibiotic binding, efflux pumps. A major challenge to counteract the development of resistance to antibiotic treatment is to get a better understanding of how bacteria react to antibiotics.

The efficiency of many antibiotics is known to be impaired by the existence of resistance mechanisms. In this study, an antibiotic resistance gene, aphII, was used to assess the impact of the level of expression of this gene on survival to an antibiotic selective pressure. aphII encodes a phosphotransferase, phosphorylating the aminoglycoside antibiotic, neomycin, impairing its ability to interact with the ribosome and thus preventing inhibition of mRMA translation by neomycin (Beck et al. 1982; Hermann 2007). The expression of this gene present on the multicopy plasmid pIJ487 (Ward et al. 1986) was put under the control of 18 promoters of varying strengths originating from a previous study 
(Seghezzi et al. 2011). These different constructs were introduced into Streptomyces lividans and survival of these different clones, exposed to a constant and rather high concentration of neomycin $(100 \mu \mathrm{g} / \mathrm{ml})$, was assessed.

Our results revealed that the relation between promoter strength (as determined by RT-PCR) and survival rate was not linear but indicated a sigmoidal correlation. A model, consistent with this behaviour, based on the well-known mechanism by which aminoglycoside antibiotics are lethal to bacteria, and how AphII counteracts this poisoning activity, was designed and discussed. This model might have a more general scope to rationalize the currently observed but never explained sigmoidal shape of classical inhibition curve obtained with linearly increasing antibiotic concentrations (Baudoux et al. 2007).

\section{Materials and methods}

\section{Bacterial strains, plasmid and media}

Streptomyces lividans TK24 strains transformed with 38 pIJ487-derived plasmids, each carrying a 300 bp DNA fragment with promoter activity of various strength were used in this study (Ward et al. 1986). The strength of these promoters was previously roughly estimated, using the replica-plating technique as described in (Lederberg and Lederberg 1952). These promoters were classified as weak, medium or strong based on their ability to allow growth of the different transformants in the presence of up to 20,50 or $100 \mu \mathrm{g} \cdot \mathrm{ml}^{-1}$ of neomycin in HT medium (Seghezzi et al. 2011). Media as well as Streptomyces manipulations were carried out according to Practical Streptomyces Genetics manual (Kieser et al. 2000). SFM was used to grow up transformants to prepare spores suspensions for quantitative estimation of survival rates.

\section{Estimation of survival rates}

Glycerol stocks of spores of the different transformants made on SFM medium were precisely titrated by plating different spore dilutions on HT agar containing $50 \mu \mathrm{g} \cdot \mathrm{ml}^{-1}$ thiostrepton. Subsequently, $10^{2}$ and $10^{3}$ spores of each transformant were spread out on HT agar plates containing $50 \mu \mathrm{g} \cdot \mathrm{ml}^{-1}$ thiostrepton only or $50 \mu \mathrm{g} \cdot \mathrm{ml}^{-1}$ thiostrepton and $100 \mu \mathrm{g} \cdot \mathrm{ml}^{-1}$ neomycin. Neomycin resistant colonies were counted after incubation for 72 hours at $30^{\circ} \mathrm{C}$ and viability rates were calculated relative to the number of colonies on neomycin free plates. These platings, done in duplicate, gave very similar viability counts and the means are shown in Figure 1.

\section{Estimation of aphll transcriptional activity using RT-PCR} RNA was extracted, using RNeasy Mini Kit from Qiagen, from selected transformants representative of each strength class and grown for $48 \mathrm{~h}$ on the surface of cellophane disks

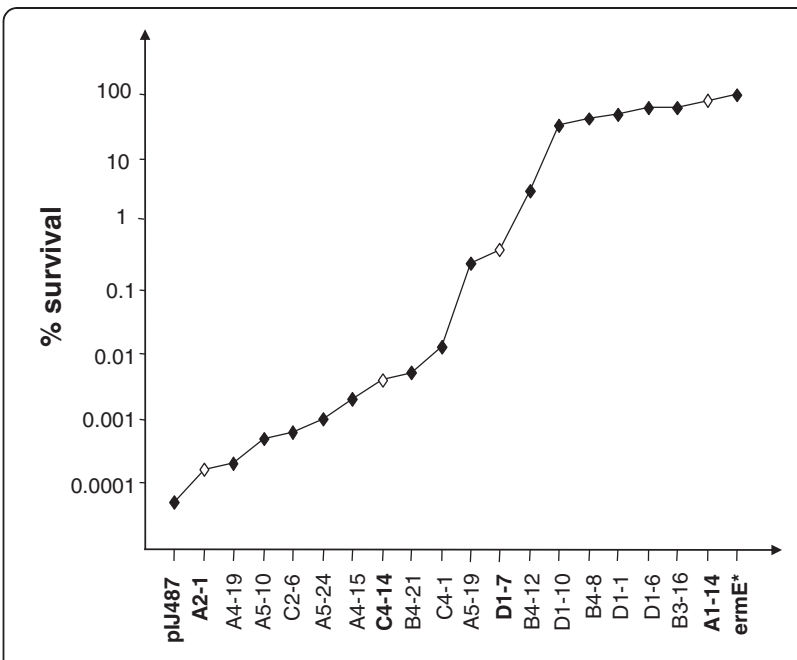

Figure 1 Survival rates conferred by promoters of different strength on HT medium containing $100 \mu \mathrm{g} \cdot \mathrm{ml}^{-1}$ neomycin. Promoters strength was initially roughly assessed by patch replica plating on HT medium containing different concentrations of neomycin. Transcriptional activity conferred by the promoters indicated in bold as well as that originating from control plasmids (promoter-less plJ487 and plJ487ermE*) were determined by RT-PCR.

laid on solid HT medium containing only $50 \mu \mathrm{g} \cdot \mathrm{ml}^{-1}$ thiostrepton.

RT PCR was performed using the OneStep RT-PCR Kit from Qiagen and the following conditions: initial denaturation at $97^{\circ} \mathrm{C}, 5 \mathrm{~min}$ followed by 25 cycles of denaturation $\left(97^{\circ} \mathrm{C}, 30 \mathrm{~s}\right)$, annealing $\left(50^{\circ} \mathrm{C}, 30 \mathrm{~s}\right)$ and extension $\left(72^{\circ} \mathrm{C}, 30 \mathrm{~s}\right)$. The absence of DNA in RNA samples was systematically checked by running a control PCR reaction made in absence of reverse transcription. Quantification of the RT-PCR signals was made using ImageQuant pixel counts in non-saturated conditions. Values were normalised on pIJ487 signals. The normalisation was done with the negative control that is the plasmid pIJ487 with no promoter cloned upstream of aphII. Some weak transcription of aphII was detected in this context.

\section{Results}

\section{Assessment of promoter strength using viability assays} and RT-PCR

An accurate quantification of promoter strength, expressed as survival rate, was carried out in the presence of $100 \mu \mathrm{g}$. $\mathrm{ml}^{-1}$ neomycin, for 38 selected clones belonging to a previously constructed bank of synthetic promoter designed for Streptomyces species and fused to the reporter gene aphII conferring resistance to neomycin. These promoters were previously roughly classified as weak, medium and strong by replicate plating (Seghezzi et al. 2011). A transformant containing pIJ487ermE* carrying the strong ermE* promoter was used as a positive control (Bibb et al. 1985). It should be stressed that in a genetically homogenous 
bacterial population, all the bacteria are not in the same physiological state and the expression of aphII (as that of any other genes) varies stochastically around a mean value (Elowitz et al. 2002; Losick and Desplan 2008). This variability explains why, even when a weak promoter is driving aphII expression, a small fraction of the bacterial population is able to resist to a high level of neomycin.

Eighteen of these transformants, representative of each class of promoter strength (weak, medium and strong) were precisely ranked according to the survival rate they conferred in the presence of $100 \mu \mathrm{g} \cdot \mathrm{ml}^{-1}$ neomycin. All colonies had approximately the same size. Each promoter was plotted against the log of the survival rate it conferred in the presence of $100 \mu \mathrm{g} \cdot \mathrm{ml}^{-1}$ neomycin. Interestingly, the resulting curve appears to be sigmoidal (Figure 1). The first and third parts represent the low and high survival rates and the central part of the curve shows an abrupt transition between these two states. We thus wondered whether the transcriptional activity of these promoters followed a similar sigmoidal pattern. To answer this question, we assessed the transcriptional activity of promoters corresponding to the three parts of the curve, using RT-PCR.

Results shown in the Figure $2 \mathrm{AB}$ indicated that the transcriptional activity between the weakest and the strongest promoter was approximately 12 fold whereas the corresponding overall increase in the survival rate was in the $10^{6}$ fold range. Similarly, the two fold increase in the level of expression between the D1-7 and A1-14 promoters led to a 200 fold increase in the survival rate. The level of gene expression was thus plotted versus the survival rates and this plotting (Figure $2 \mathrm{C}$ ) indicated a non-linear, roughly sigmoidal, correlation between the level of gene expression and the survival rate. We thus proposed an explanatory model that rationalises the sigmoidal pattern of resistance to neomycin observed in relation with the level of aphII gene expression and with the concentration of neomycin used.

\section{The model}

Our study revealed a sigmoidal correlation between the level of aphII gene expression and the survival rates, in the presence of neomycin $100 \mu \mathrm{g} \cdot \mathrm{ml}^{-1}$ (Figure 2C). This behaviour is reminiscent of a system containing a positive feedback loop leading to two different states, the transition between the two states being very abrupt (Kaufman et al. 2007; Mehra et al. 2008; Mitrophanov and Groisman 2008). In our case, the positive feedback loop would be constituted by one positive and two negative interactions (Figure 3). Positive interaction (PI): the ribosomes are involved in protein synthesis. Negative interaction (NI1): neomycin poisons the ribosomes leading to an inhibition of protein synthesis, including that

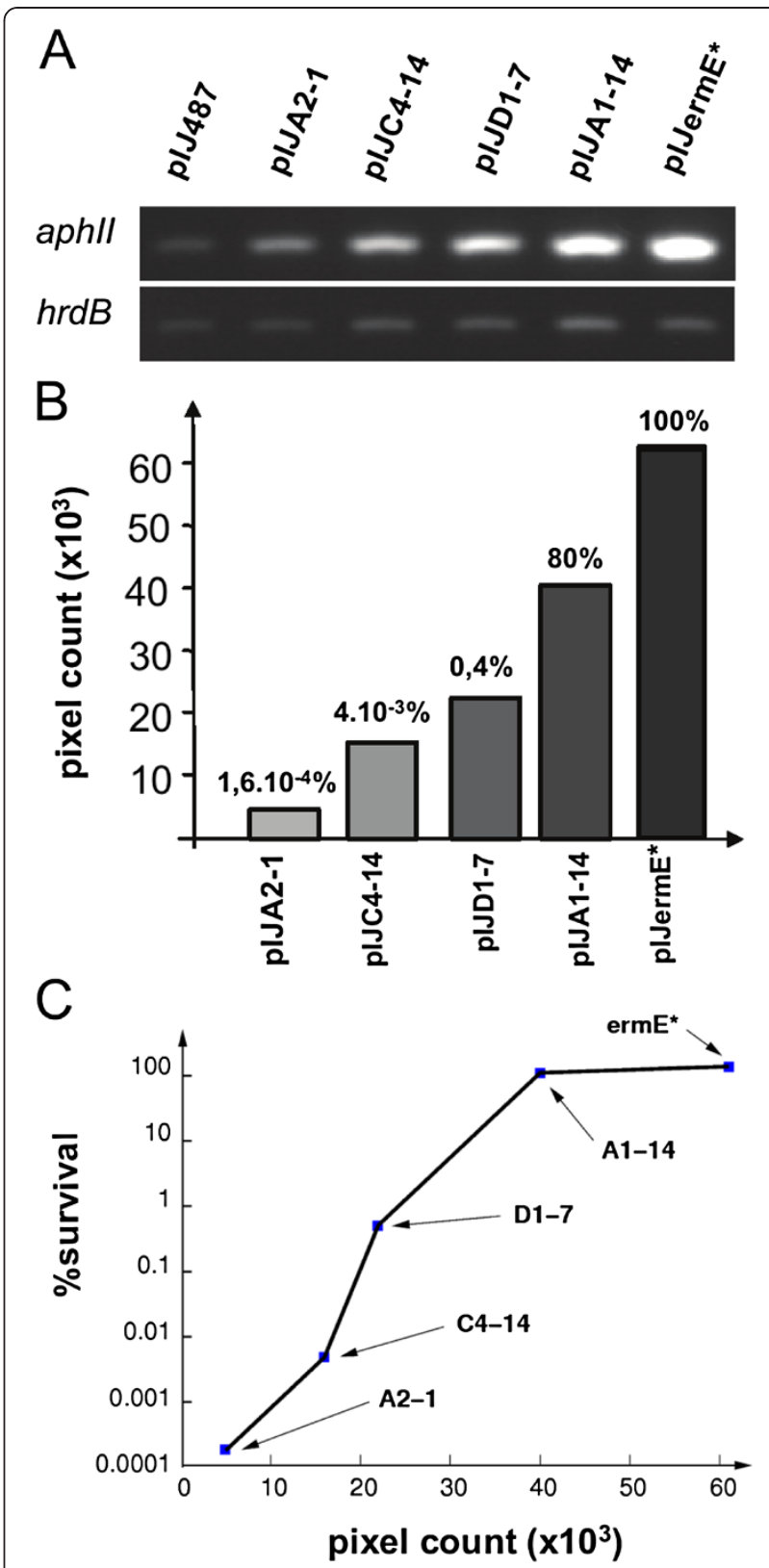

Figure $\mathbf{2}$ Correlation between the level of transcription of the aphll gene and the survival rates of different transformants in the presence of $100 \mu \mathrm{g} \cdot \mathrm{ml}^{-1}$ neomycin. (A) Assessment of the transcriptional activity of aphll in S. Lividans driven by the promoters highlighted in bold in Figure 1 and the control plasmids, plJ487 and plJ487ermE* , using RT-PCR. Clones were ordered from the left to the right according to increasing promoter strength. (B) Quantification of the RT-PCR signals was made using ImageQuant pixel counts in non-saturated conditions. Values were normalized on plJ487 signals. Survival rates corresponding to each clone are indicated above each histogram. (C) Plotting of the quantification of the RT-PCR products of the different clones versus their corresponding survival rates in presence of $100 \mu \mathrm{g} \cdot \mathrm{ml}^{-1}$ neomycin. 
of AphII. Negative interaction (NI2): AphII inactivates neomycin. These two negative interactions and the positive one generate a positive circuit. When the expression of aphII is low, AphII is not very abundant so most of neomycin is active and a lot of ribosomes are poisoned. This poisoning leads to a further reduction of AphII and

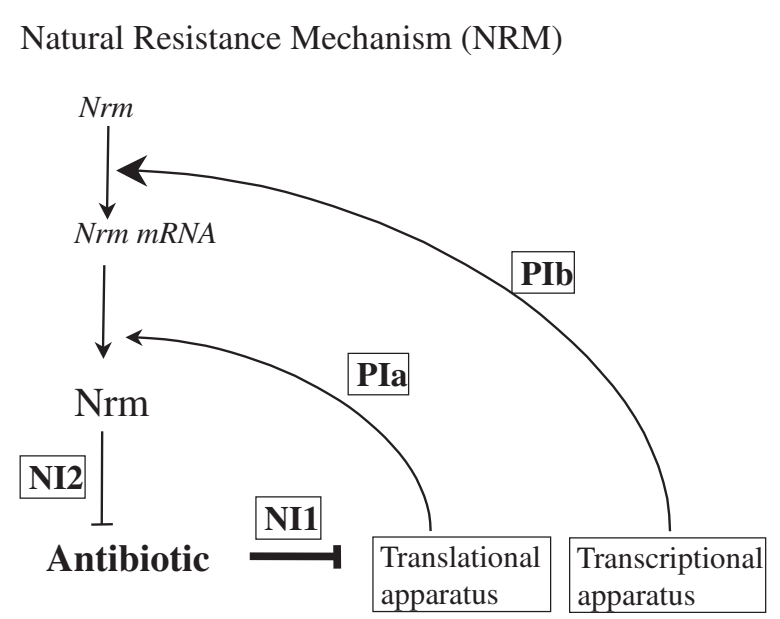

Figure 4 Model of natural mechanisms of resistance against antibiotics targeting the transcriptional and/or the translational apparatus. The transcriptional and the translational apparatus are involved in protein synthesis (positive interactions Pla, Plb). The lethal effect of an antibiotic relies on its ability to inhibit transcription or translation (first negative interaction, NI1) including that of a gene encoding a protein neutralizing somehow the poisoning effect of the antibiotic (second negative interaction, NI2) The positive and the two negative interactions generate a positive circuit that leads to the establishment of a sigmoidal pattern of resistance to the antibiotic according to the level of expression of the gene responsible for the natural resistance mechanism. thus to low survival rates (State 1). Conversely, when the expression of aphII is high, AphII is abundant, most neomycin is inactivated so more ribosomes are functional leading to a further enhancement of aphII mRNA translation leading to high survival rates (State 2). A small variation of gene expression around a specific threshold leads to an abrupt switch from one state to the other. Experimentally, the level of expression of the D1-7 promoter was shown to be close to this threshold.

\section{Discussion}

The aphII gene is the most extensively used reporter system in Streptomyces, however, this useful system was sometimes blamed for some non-understood paradoxical behaviour. The dynamics of the system, as revealed by our study, can explain these paradoxes. Consequently, the aphII gene should thus be used with caution to accurately assess promoter strength, since a small variation of gene expression around a specific threshold might lead to a huge change in the pattern of resistance to neomycin.

Furthermore, our study suggests that it might be sufficient to reduce (and not totally preclude) the expression of a resistance gene just under a certain threshold to greatly enhance the killing efficiency of the corresponding antibiotic. That is why nowadays even imperfect inhibitors of transcription and/or translation are sometimes associated in prescription to overcome some reluctant antibiotic resistant strains.

At last, it is noteworthy that the sigmoidal shape of our curve is reminiscent of that of classical inhibition curve obtained with linearly increasing concentration of antibiotics (Baudoux et al. 2007; Mattie 2000). This similarity suggests that related processes might take place in a 
natural strain. Any natural strain, in which no exogenous antibiotic resistance gene was introduced, does possess more or less efficient active antibiotic resistance processes. These processes might include efflux of the antibiotic via multidrug efflux pumps (Nikaido 2009), built-in target (Criswell et al. 2006; Long et al. 2009) or antibiotic modifications or proteic systems that might counteract the detrimental consequences induced by the antibiotic such as the generation of oxidative stress etc. (Hassett and Imlay 2007; Kohanski et al. 2007; Shin et al., 2011). Since all these resistance processes involve genes that should be transcribed and mRNA that should be translated, it is not unreasonable to think that any antibiotic targeting the transcriptional or translational apparatus would give rise to a positive circuit resulting in a sigmoidal response (Figure 4). Our model thus might provide a sound explanation for the sigmoidal shape of the inhibition curves that has been repeatedly observed but was never explained.

\section{Competing interests}

The authors declare that they have no competing interests.

\section{Acknowledgements}

This work was supported by the European program ACTINOGEN (http://www.swan.ac.uk/ils/Research/BioMed/ActinoGen/), the Centre National de la Recherche Scientifique (http://www.cnrs.fr/), the University Paris Sud (http://www.u-psud.fr) and the Pôle de Recherche et d'Enseignement supérieur UniverSud Paris (http://www.universud-paris.fr).

\section{Author details}

${ }^{1}$ Institut de Génétique et Microbiologie, UMR8621 CNRS Université Paris-Sud, Orsay 91405, France. 'Laboratoire de Recherche en Informatique, Université Paris-Sud, UMR8623 CNRS, and INRIA Saclay, Orsay 91405, France.

Received: 30 January 2013 Accepted: 4 February 2013

Published: 8 February 2013

\section{References}

Allen HK, Donato J, Wang HH, Cloud-Hansen KA, Davies J, Handelsman J (2010) Call of the wild: antibiotic resistance genes in natural environments. Nat Rev Microbiol 8:251-259

Alonso A, Sanchez P, Martinez JL (2001) Environmental selection of antibiotic resistance genes. Environ Microbiol 3:1-9

Baudoux P, Bles N, Lemaire S, Mingeot-Leclercq MP, Tulkens PM, Van Bambeke F (2007) Combined effect of $\mathrm{pH}$ and concentration on the activities of gentamicin and oxacillin against Staphylococcus aureus in pharmacodynamic models of extracellular and intracellular infections. J Antimicrob Chemother 59:246-253

Beck E, Ludwig G, Auerswald EA, Reiss B, Schaller H (1982) Nucleotide sequence and exact localization of the neomycin phosphotransferase gene from transposon Tn5. Gene 19:327-336

Bibb MJ, Janssen GR, Ward JM (1985) Cloning and analysis of the promoter region of the erythromycin resistance gene (ermE) of Streptomyces erythraeus. Gene 38:215-226

Butler MS, Cooper MA (2012) Screening strategies to identify new antibiotics. Curr Drug Targets 13:373-387

Criswell D, Tobiason VL, Lodmell JS, Samuels DS (2006) Mutations conferring aminoglycoside and spectinomycin resistance in Borrelia burgdorferi. Antimicrob Agents Chemother 50:445-452

Davies J, Davies D (2010) Origins and evolution of antibiotic resistance. Microbiol Mol Biol Rev 74:417-433

Elowitz MB, Levine AJ, Siggia ED, Swain PS (2002) Stochastic gene expression in a single cell. Science 297:1183-1186

Forsberg KJ, Reyes A, Wang B, Selleck EM, Sommer MO, Dantas G (2012) The shared antibiotic resistome of soil bacteria and human pathogens. Science 337:1107-1111
Gyssens IC (2011) Antibiotic policy. Int J Antimicrob Agents 38(Suppl):11-20 Hassett DJ, Imlay JA (2007) Bactericidal antibiotics and oxidative stress: a radical proposal. ACS Chem Biol 2:708-710

Hermann T (2007) Aminoglycoside antibiotics: old drugs and new therapeutic approaches. Cell Mol Life Sci 64:1841-1852

Kaufman M, Soule C, Thomas R (2007) A new necessary condition on interaction graphs for multistationarity. J Theor Biol 248:675-685

Kieser T, Bibb MJ, Buttner MJ, Chater KF, Hopwood DA (2000) Practical Streptomyces Genetics. John Innes centre, Norwich Research Park, Norwich

Kohanski MA, Dwyer DJ, Hayete B, Lawrence CA, Collins JJ (2007) A common mechanism of cellular death induced by bactericidal antibiotics. Cell 130:797-810

Lederberg J, Lederberg EM (1952) Replica plating and indirect selection of bacterial mutants. J Bacteriol 63:399-406

Levy SB (1998) The challenge of antibiotic resistance. Sci Am 278:46-53

Levy SB (2002) The Antibiotic Paradox: How the Misuse of Antibiotics Destroys Their Curative Power. Da Capo Press, Perseus Publishing, Cambridge, MA, USA. ISBN 0-7382-0440-4

Long KS, Poehlsgaard J, Hansen LH, Hobbie SN, Bottger EC, Vester B (2009) Single $23 \mathrm{~S}$ rRNA mutations at the ribosomal peptidyl transferase centre confer resistance to valnemulin and other antibiotics in Mycobacterium smegmatis by perturbation of the drug binding pocket. Mol Microbiol 71:1218-1227

Losick R, Desplan C (2008) Stochasticity and cell fate. Science 320:65-68

Mahajan GB, Balachandran L (2012) Antibacterial agents from actinomycetes - a review. Front Biosci (Elite Ed) 4:240-253

Martinez JL (2008) Antibiotics and antibiotic resistance genes in natural environments. Science 321:365-367

Mattie H (2000) Antibiotic efficacy in vivo predicted by in vitro activity. Int Antimicrob Agents 14:91-98

Mehra S, Charaniya S, Takano E, Hu WS (2008) A bistable gene switch for antibiotic biosynthesis: the butyrolactone regulon in Streptomyces coelicolor. PLOS One 3:e2724

Mitrophanov AY, Groisman EA (2008) Positive feedback in cellular control systems. Bioessays 30:542-555

Nikaido H (2009) Multidrug resistance in bacteria. Annu Rev Biochem 78:119-146

Seghezzi N, Amar P, Koebmann B, Jensen PR, Virolle MJ (2011) The construction of a library of synthetic promoters revealed some specific features of strong Streptomyces promoters. Appl Microbiol Biotechnol 90:615-623

Shin JH, Singh AK, Cheon DJ, Roe JH (2011) Activation of the SoxR regulon in Streptomyces coelicolor by the extracellular form of the pigmented antibiotic actinorhodin. J Bacteriol 193:75-81

Ward JM, Janssen GR, Kieser T, Bibb MJ, Buttner MJ (1986) Construction and characterisation of a series of multi-copy promoter-probe plasmid vectors for Streptomyces using the aminoglycoside phosphotransferase gene from Tn5 as indicator. Mol Gen Genet 203:468-478

doi:10.1186/2191-0855-3-13

Cite this article as: Seghezzi et al:: Novel insights regarding the sigmoidal pattern of resistance to neomycin conferred by the aphll gene, in Streptomyces lividans. AMB Express 2013 3:13.

\section{Submit your manuscript to a SpringerOpen ${ }^{\odot}$ journal and benefit from:}

- Convenient online submission

- Rigorous peer review

- Immediate publication on acceptance

- Open access: articles freely available online

- High visibility within the field

- Retaining the copyright to your article

Submit your next manuscript at $>$ springeropen.com 